\title{
BMJ Open Cognitive control and daily affect regulation in major depression and borderline personality disorder: protocol for an experimental ambulatory assessment study in Berlin, Germany
}

\author{
Lars Schulze, ${ }^{1}$ Paul-Christian Bürkner, ${ }^{2}$ Julian Bohländer, ${ }^{1}$ Ulrike Zetsche ${ }^{1}$
}

To cite: Schulze L, Bürkner P-C, Bohländer J, et al. Cognitive control and daily affect regulation in major depression and borderline personality disorder: protocol for an experimental ambulatory assessment study in Berlin, Germany. BMJ Open 2018;8:e22694. doi:10.1136/ bmjopen-2018-022694

- Prepublication history for this paper is available online. To view these files, please visit the journal online (http://dx.doi. org/10.1136/bmjopen-2018022694).

Received 1 March 2018 Revised 22 June 2018 Accepted 16 August 2018

Check for updates

(C) Author(s) (or their employer(s)) 2018. Re-use permitted under CC BY-NC. No commercial re-use. See rights and permissions. Published by BMJ.

${ }^{1}$ Department of Clinical Psychology and Psychotherapy, Freie Universität Berlin, Berlin, Germany

${ }^{2}$ Department of Statistics, Faculty of Psychology, University of Münster, Münster, UK

Correspondence to Dr Ulrike Zetsche; u.zetsche@fu-berlin.de

\section{ABSTRACT}

Introduction Affective disturbances and difficulty in affect regulation are core features of major depressive disorder (MDD) as well as borderline personality disorder (BPD). Whereas depressed individuals are characterised by affective inertia, individuals with BPD are characterised by affective instability. Both groups have been found to use more maladaptive affect regulation strategies than healthy controls. Surprisingly, however, there have been hardly any studies directly comparing these two disorders to disentangle shared and disorder-specific deficits in affective dynamics and affect regulation. Furthermore, theoretical models link deficits in affect regulation to deficits in cognitive control functions. Given that individuals with MDD or BPD are both characterised by impairments in cognitive control, the present study will further examine the link between individual differences in cognitive control and disturbances in affect dynamics and regulation in the daily life of individuals with MDD or BPD.

Methods and analyses We will use a smartphone application to assess negative and positive affect as well as affect regulation strategies at eight times a day for 7 days. We will further employ four computerised tasks to assess two cognitive control functions, namely interference control and discarding irrelevant information from working memory. Our hypotheses will be tested using a multimethod approach. Power analyses determined a sample size of 159 (53 MDD, $53 \mathrm{BPD}, 53$ controls) to detect medium effect sizes.

Ethics and dissemination Ethics approval has been obtained from the Freie Universität Berlin. Data collection started in January 2017 and will last until the end of 2018. Results will be disseminated to relevant psychotherapeutic and patient communities in peer-reviewed journals, and at scientific conferences.

\section{INTRODUCTION}

Affective disturbances are common among most mental disorders. In search of causes for these affective disturbances, impairments in the regulation of affective states have become a major interest in clinical psychology. The most prominent and generalised
Strengths and limitations of this study

- Real-time assessment of affect dynamics and affect regulation in daily life.

- Assessing two prominent affective disorders (borderline personality disorder and major depressive disorder) and a control group.

- Linking the use and effectiveness of affect regulation strategies to individual differences in cognitive control functions (ie, discarding of previously relevant information, interference control).

- Limitations: cross-sectional design, mainly self-report measures of affect regulation.

impairments in affect regulation (AR) are found in individuals with major depressive disorder (MDD) or borderline personality disorder (BPD). ${ }^{12}$ Although there is growing research examining abnormalities in the use and effectiveness of AR strategies, hardly any study has directly compared these two disorders to disentangle shared and disorder-specific deficits in affect regulation. In addition, theoretical models have linked effective AR to cognitive control functions (for reviews, see Joorman and D'Avanzato and Ochsner and Gross $^{34}$ ). Identification of abnormalities in affect regulation and its underlying cognitive mechanisms thus represents an important step in developing interventions to address deficits in affect regulation in these disorders.

The following paragraphs give an overview of previous findings on the use and effect of the three most researched AR strategies, that is, rumination, suppression, and reappraisal in MDD and BPD, while highlighting important questions that have as yet remained unanswered.

\section{Affect regulation in depression}

Affective disturbances in depression are characterised by both the experience of sustained 
negative affect (ie, affective inertia ${ }^{5}$ ) as well as difficulty experiencing positive affect. ${ }^{6}$ To gain a better understanding of these affective disturbances, recent research has focused on the way depressed individuals attempt to regulate their affect. Results revealed that depressed individuals as compared with healthy controls show a greater use of putatively maladaptive affect regulation strategies (for a review, see Aldao $e t a l^{7}$ ). In this context, rumination has been identified as a particularly detrimental response to negative affect. ${ }^{2}$ Rumination involves recurrent negative thoughts focused on one's depressive symptoms and the causes, meaning and consequences of these symptoms. ${ }^{8}$ Rumination in response to negative affect has been shown to intensify negative affect, increase negative memory recall, impair social problem solving and ultimately enhance the risk for the onset of new depressive episodes (for a review, see Watkins ${ }^{9}$ ). Another maladaptive regulation strategy that has been linked to depression is the suppression of one's affect. Currently depressed as well as remitted depressed individuals have been found to suppress their affective response to a greater degree than non-depressed individuals. ${ }^{710}$ Although intended to reduce negative affect, suppression has been found to increase negative affect. ${ }^{10} 11$ On the other hand, evidence also suggests that depressed individuals are less likely to use AR strategies that are beneficial in healthy individuals. ${ }^{12}$ Cognitive reappraisal has been shown to be a particularly effective means of AR. ${ }^{13}$ Reappraisal involves changing the meaning of a situation in order to alter the affect that follows. ${ }^{14}$ In a recent meta-analysis, decreased habitual use of reappraisal has been associated with depressive symptoms. ${ }^{7}$ Thus, evidence suggests that depression is associated with more frequent use of maladaptive AR strategies, such as rumination or suppression, and less frequent use of adaptive strategies, such as reappraisal. Please note that all hypotheses of the present project focus on these three most-researched AR strategies (ie, rumination, suppression, reappraisal). When examining group differences in the use of specific AR strategies, however, it is important to control for overall AR strategy use. ${ }^{15}$ For this purpose, we also assess other widely used strategies, namely distraction, acceptance and social sharing. Whereas findings on the association between distraction and depression are inconclusive, ${ }^{2}$ there are hardly any studies on the association of acceptance or social sharing and depression. Thus, we do not formulate any specific hypotheses regarding these AR strategies.

In addition, there is evidence suggesting that depressed individuals are not able to employ putatively adaptive AR strategies as effectively as healthy individuals. Joormann and colleagues, for example, demonstrated that currently depressed compared with healthy individuals were not able to use positive memories to repair a negative affective state. ${ }^{16}$ Further research found that higher levels of depressive symptoms were associated with lower reappraisal ability under high levels of stress. ${ }^{17}$ Thus, strategies that are effective in regulating negative affect in healthy individuals may not be as effective in the regulation of negative affect in currently depressed individuals.

\section{Affect regulation in borderline personality disorder}

The affective disturbance that is 'at the core of borderline pathology $^{18}$ is a pronounced instability of emotions. ${ }^{19}$ Pivotal to the understanding of this pronounced instability are abnormalities in the processing and regulation of affective responses. ${ }^{2021}$

Regarding affect regulation, evidence suggests a more pronounced use of affect suppression in BPD. ${ }^{22}{ }^{23}$ In addition, heightened levels of rumination have been reported in BPD as compared with healthy individuals. ${ }^{2425}$ Students with pronounced traits of borderline personality demonstrate a generally increased use of adaptive as well as maladaptive AR strategies to regulate affective states. ${ }^{26}$

Only recently, studies have begun to examine the effectiveness of AR strategies in BPD. In contrast to findings in healthy individuals and patients with MDD, the suppression of affective responses was found to decrease negative affect and to attenuate impulsive behaviour. ${ }^{27}$ Recent findings provided further support that affect suppression may have an adaptive function in $\mathrm{BPD} .{ }^{28}$ In addition, findings suggest that individuals with BPD as compared with healthy controls use cognitive reappraisal less efficiently to attenuate negative affect. ${ }^{29} 30$ This might be due to difficulties in the generation and implementation of alternative appraisals of affect-generating stimuli. ${ }^{20}$

\section{Affect regulation and cognitive control deficits}

The mechanisms underlying impairments in effective affect regulation are not yet well researched. Several researchers have suggested that cognitive control functions play an important role in effective affect regulation. ${ }^{34}$ Affective states are associated with the activation of affect congruent cognitions in working memory. The ability to control affective contents in working memory may thus be essential for effective affect regulation. It is important to note that cognitive control is not a unitary construct but consists of several components, such as response inhibition, discarding of no longer relevant material from working memory or interference control (ie, resistance to distractor interference). ${ }^{31} 32$

Impairments in cognitive control have been generally linked to both BPD $^{33-36}$ and depression symptoms. ${ }^{37-40}$ Importantly, impairments in cognitive control have also been directly linked to disturbances in affect regulation: more frequent $u$ se of rumination has been related to difficulty discarding no longer relevant material from working memory, ${ }^{41}$ whereas more frequent use of suppression has been linked to impairments in interference control of negative material. ${ }^{42}$ In addition, less frequent use of reappraisal may be related to difficulty in interference control. ${ }^{424}$

Fewer studies have assessed the role cognitive control plays in the effectiveness of AR strategies. First evidence implies that deficits in the ability to discard previously relevant information from working memory confine 
the benefits of reappraisal and increase the detrimental effects of rumination. ${ }^{1544}$ However, this has not yet been assessed in a clinical sample. It will therefore be crucial to examine the link between differences in the cognitive control of affective material and the effectiveness of daily affect regulation in clinical samples.

\section{Research questions and hypotheses}

The present project combines the assessment of daily affective dynamics, daily affect regulation strategies and cognitive control abilities in individuals with MDD, individuals with BPD and healthy controls. This design is a compelling framework to examine the following research questions:

\section{Affective dynamics}

Research question: Do healthy controls, individuals with MDD and individuals with BPD differ in their affective dynamics (ie, affective inertia, affective instability) in daily life?

Hypotheses: We expect a main effect of group on each measure of affect dynamics. Specifically, we expect that individuals with BPD show more affective instability than individuals with MDD or healthy controls, when controlling for affective variability. ${ }^{5}$ Further, we expect that individuals with MDD or BPD show higher affective variability than healthy controls, even after controlling for inertia. Finally, we expect that individuals with BPD show less affective inertia than individuals with MDD or healthy controls, when controlling for affective variability.

\section{Use of affect regulation strategies}

Research question: Do healthy controls, individuals with MDD and individuals with BPD differ in their habitual use of different affect regulation strategies?

Hypotheses: First, we expect a main effect of group on general intensity of affect regulation. That is, we expect that individuals with BPD or MDD generally employ affect regulation strategies more often than healthy controls. Second, we expect an interaction between group and kind of strategy. ${ }^{722} 2426$ That is, we expect that individuals with BPD or MDD select rumination or suppression more often than reappraisal. In contrast, we expect that healthy controls select reappraisal more often than rumination or suppression.

\section{Effect of affect regulation strategies on affect}

Research question: Does the effectiveness of affect regulation strategies differ between healthy controls, individuals with MDD and individuals with BPD?

Hypotheses: We expect an interaction effect between group and kind of strategy on affect ratings. Specifically, we expect that rumination intensity assessed as time $t$ will be associated with higher negative affect at time $t$ when controlling for negative affect at $t-1$ in individuals with MDD or BPD than in healthy controls. ${ }^{45}{ }^{46}$ Note that rumination assessed at time $t$ reflects the intensity in the interval between time $t-1$ and time $t$.
The intensity of suppression assessed at time $t$ will be associated with lower negative affect at time $t$ when controlling for negative affect at $t-1$ in individuals with $\mathrm{BPD},{ }^{26}{ }^{28}$ but not in individuals with MDD and healthy controls. ${ }^{1011}$

The intensity of reappraisal assessed at time $t$ will be associated with more negative affect at time $t$ when controlling for negative affect at $t-1$ in individuals with BPD or MDD as compared with healthy controls. ${ }^{172029}$

\section{Group differences in cognitive control}

Research question: Do healthy controls, individuals with MDD and individuals with BPD differ in their ability to control affective material in working memory?

Hypotheses: We expect an interaction effect between group and experimental condition on response latencies. Specifically, we expect that individuals with MDD or BPD as compared with healthy controls show impairments in interference control of affective stimuli, reflected in slower response latencies in experimental as compared with control trials. ${ }^{34} 47$

Similarly, we expect that individuals with MDD as compared with healthy controls show impairments in discarding no longer relevant negative material from working memory, reflected in slower response latencies in experimental as compared with control trials. ${ }^{38}$

\section{Cognitive control and affect regulation}

Research question: Are impairments in cognitive control functions related to differences in the use or effectiveness of affect regulation strategies?

Hypotheses: We expect an interaction between the respective cognitive control index and kind of strategy on intensity ratings (ie, strategy use). That is, we expect that individual differences in discarding affective material from working memory will be negatively associated with more frequent use of rumination. ${ }^{38414}$

In addition, we expect that individual differences in interference control will be negatively associated with using suppression and positively associated with using reappraisal. $^{38}$

We further expect an interaction between the respective cognitive control index and kind of strategy on negative affect ratings at time $t$. That is, we expect that individual differences in discarding affective material from working memory when using rumination will be associated with higher negative affect at time $t$ when controlling for negative affect at time $t-1 .{ }^{15}{ }^{44}$ Further, we expect that individual differences in discarding affective material from working memory when using reappraisal will be associated with less negative affect at time $t$ when controlling for negative affect at time $t-1 .{ }^{15} 44$

Note, that the number of studies directly comparing individuals with MDD and BPD regarding affective dynamics, affect regulation strategies or cognitive control abilities is very limited. Thus, the literature only allows to formulate specific hypotheses on differences between the clinical groups and the control group. It will be intriguing 
to examine differences and similarities between individuals with MDD and individuals with BPD in the assessed variables.

\section{Additional research questions}

The present research project allows to investigate several additional research questions that will be presented below:

Heart rate variability and affect regulation: Thayer and Lane ${ }^{48}$ proposed that heart rate variability reflects a psychophysiological index of affect regulation capacity. Indeed, several studies illustrated that individuals with low resting vagally mediated heart rate variability (vmHRV) have difficulties with affect regulation. ${ }^{49}{ }^{50}$ Accordingly, lower HRV has been reported for individuals with BPD and MDD, ${ }^{51}{ }^{52}$ but to date no study directly assessed the role of vmHRV on affective dynamics or affect regulation. In this study, we include a resting-state assessment of HRV to examine this question.

Physical activity: There is considerable evidence that people feel better after being physically active.$^{53}$ However, these findings are almost entirely based on interventional, between-person designs. Hence, it is unclear whether these findings translate into daily life. It will thus be interesting to examine the associations between daily physical activity and daily affective states. ${ }^{54}$ For these reasons, participants of our study are asked to wear an accelerometer during the ambulatory assessment phase.

Expectation and recall biases of affective states: Depressive symptoms are associated with pronounced biases in the expectation and recall of affective states. ${ }^{55}$ Notably, such biases also affect the choice and implementation of emotion regulation strategies. ${ }^{56} 57$ These processes, however, have been primarily investigated in non-clinical samples. In this study, depressed individuals and individuals with BPD are asked to predict their average affect, sleep and affect regulation before the ambulatory assessment phase. After the ambulatory assessment phase, they have to recall their average weekly affect, sleep and affect regulation.

\section{METHODS AND ANALYSES \\ Participants}

The present research project includes three groups of participants: individuals with BPD, individuals with current MDD and healthy control participants. The sample size is targeted at 53 participants per group (for details see power analysis).

\section{General inclusion and exclusion criteria}

Participants will be between age 18 and 65 years and speak German as their native language (due to verbal demands in the experimental tasks). Participants need to provide written informed consent for participation in the study. Participants will be excluded if they

- Are pregnant,
- Report of severe head trauma or any known neurological diseases,

- Report any past or present psychotic symptoms,

- Meet criteria for bipolar disorder or any psychotic disorder,

- Meet criteria for substance dependency within the last 12 months.

Patients taking psychotropic medication will not be excluded. However, there must be no change in medication for at least 4 weeks prior to as well as during the entire assessment period. Medication type and dose will be assessed. Inpatients will not be included in the study.

\section{Major depressive disorder (MDD) group}

Participants included in the MDD group will meet Diagnostic and Statistical Manual of Mental Disorders (DSMIV) criteria for a current major depressive episode. The duration of the current episode as well as the number of past episodes will be assessed but will not be decisive for inclusion into the study. Due to high rates of comorbidity among MDD and other mood and anxiety disorders, individuals with a comorbid mood (except bipolar disorders) or anxiety disorder will be included. Depressed individuals meeting more than two DSM-IV criteria for BPD will be excluded.

\section{Borderline personality disorder (BPD) group}

Participants included in the BPD group will meet DSM-IV criteria for borderline personality disorder. Due to high rates of Axis-I and Axis-II comorbidity in BPD, presence of comorbid disorders will be allowed for study inclusion with the exception of a current major depressive episode, substance dependency within the last 12 months, bipolar or psychotic disorders. To control for the influence of total symptom severity, all analyses on group differences in affect regulation or cognitive control will be repeated including the Brief Symptom Inventory (BSI) total score as a covariate.

\section{Healthy control group}

Participants included in the healthy control group have to be free of any past or present mental disorder according to DSM-IV criteria. The absence of any mental disorder will be confirmed by Structured Clinical Interview for DSM (SCID-I) and SCID-II interviews. Participants in the control group will be free of any psychotropic medication. Furthermore, control participants will be excluded if they meet more than two DSM-IV criteria for BPD or any of the two cardinal DSM-IV criteria for MDD.

\section{Recruitment}

Individuals with MDD or BPD will be recruited through advertisements posted at cooperating counselling institutions, various sites within the community and in online newspapers. Healthy control participants will be recruited through postings at various sites within the community and in online newspapers.

In addition, depressed participants will be recruited from the local outpatient clinic at Freie Universität Berlin 
(Head: Professor Dr Babette Renneberg). Participants with BPD will also be recruited at the Department for Psychiatry and Psychotherapy at Charité Berlin (Head: Professor Dr Stefan Röpke).

\section{Assessment of psychopathology}

All participants will be interviewed using the Structured Clinical Interview for DSM-IV Axis-I ${ }^{58}$ and Axis-II disorders. ${ }^{59}$ Diagnostic interviews will be conducted by trained interviewers.

The following instruments will assess disorder-specific and general psychopathology: The Beck Depression Inventory II (BDI-II ${ }^{6061}$; the Borderline Symptom List (BSL-23) $^{62}$; the Brief Symptom Inventory (BSI) ${ }^{63} 64$; the German version of the 10-item Response Style Questionnaire (RSQ) ${ }^{65}$ Finally, the German version of the Positive and Negative Affect Schedule (PANAS) ${ }^{66}$ as well as the Dissociative Tension Scale (DSS-4) ${ }^{67}$ will be used to assess mood fluctuations and dissociative states in the laboratory sessions.

In addition, all individuals with BPD or MDD will be asked about any current and/or past psychotherapy.

\section{Ambulatory assessment of daily affect and affect regulation}

All participants receive a smartphone including an app for ambulatory assessment. Participants will be instructed to go on with their daily activities and respond to several questions when indicated by a beep. The smartphone app will be individually programmed to beep eight times a day for seven consecutive days with the daily sampling period comprising 12 hours. The sampling period will be divided into eight time blocks of equal length and the auditory signal will occur pseudorandomly within each time block, with a minimum of 1 hour between beeps. ${ }^{15}$ Responses will be time-stamped by the software.

Following each prompt, participants will indicate on a scale from 1 (not at all) to 7 (very much) how angry, anxious, ashamed, cheerful, depressed, happy and tense they feel (ie, 'How did you feel just before the beep?'). To examine whether groups differ in the frequency may have problems in correctly identifying specific negative emotions, only the average score across all negative affect ratings (ie, angry, anxious, ashamed, depressed, tense) will be used. Next, participants will be asked to indicate on a scale from 1 (not at all) to 7 (very much) how much they used the following strategies since the last beep: rumination ('I thought over and over again about a situation or my feelings'; 'How negative were these thoughts?'), suppression ('I controlled my emotion by not showing them'), reappraisal ('I have thought about the situation in a different way'). To control for overall degree of AR strategy use, ${ }^{26}$ participants are also asked about the implementation of other widely used or disorder-relevant AR strategies. ${ }^{1868}$ These are acceptance ('I accepted the situation and/or my situation'), distraction ('I found an activity to keep myself busy and distracted') and social sharing ('I found someone to talk to about my feelings'). In addition, individuals with a history of self-injurious behaviour will be asked how much they felt an urge to injure themselves. At the first daily beep, all participants will be asked to indicate on a scale from 1 (not at all) to 7 (very well) how well they slept last night.

Participants will receive an extra incentive for responding to more than $90 \%$ of beeps.

\section{Assessment of cognitive control}

Discarding of no longer relevant information from working memory Working Memory Selection Task (WMST)

The WMST assesses the ability to discard no longer relevant affective information from working memory. ${ }^{38}$

Each trial of the WMST consists of three consecutive displays: a learning display, a cue display and a probe display. On the learning display, participants are presented with two rows of three words each, one row printed in red and the other row printed in blue. Participants are instructed to memorise all six words. On the following cue display, a red or blue frame is presented indicating which row of words will be relevant for the upcoming response. Participants are instructed to keep only the relevant set in mind and disregard the other three words. Finally, on the probe display, a probe word is presented and participants are asked to decide whether or not the probe is from the relevant word set.

The probe may either be a word from the relevant word set (relevant probe), a word that participants had to learn but were then asked to forget (suppress probe), or a new word that had not been presented before (novel probe). Thus, participants have to reject both suppress probes and novel probes. It has been shown that participants take longer to reject a suppress probe compared with a novel probe ${ }^{69}$ and it has been suggested that this difference in reaction times reflects the residual activation of the no-longer-relevant suppress word. Thus, the ability to discard no longer relevant material from working memory is measured by reaction times (RTs) to suppress probes compared with novel probes. In the present version of the task, on critical trials, the red and blue rows of words include either only positive or only negative words, and the two rows always differ in valence. Thus, here we will compare the ability to discard irrelevant negative or positive information, respectively. All word stimuli are taken from the Berlin Affective Word List Reloaded (BAWL-R) ${ }^{70}$

\section{Removal and Updating Task (RUT)}

The RUT is based on a letter updating task ${ }^{71}$ adapted by Chang et $_{\text {al. }}{ }^{72}$ It measures the ability to remove no longer relevant affective contents from working memory.

Each trial begins with the presentation of three words in three frames for $3000 \mathrm{~ms}$ and participants are asked to memorise the words. Next, the words disappear and a variable number of updating steps follows. At each updating step, one of the three words is cued for removal, indicated by the respective frame turning into red colour. Then, a new word is presented in the cued frame and participants are asked to replace in mind the memorised word with 
the new word. Participants indicate the completion of their updating process by key-press. The reaction time between the presentation of the new word and participants' key-press serves as dependent variable.

Importantly, the time between the removal cue and the presentation of the new word is varied ("cuetarget interval', CTI). In long CTI conditions ( $1500 \mathrm{~ms})$, the CTI allows for a complete removal process, so that the reaction time between the presentation of the new word and participants' key press only reflects the encoding of the new word. In the short CTI condition $(200 \mathrm{~ms})$, the CTI does not allow for a complete removal process, so that the reaction time reflects the removal process and the encoding of the new word. Thus, the measure of an individual's removal speed is the comparison between RTs in trials with short and long CTI. Indices reflecting the removal of negative or positive words can be computed. All word stimuli are taken from the BAWL-R.

To control for general updating ability, participants also complete a neutral version of the $\mathrm{RUT}^{71}$ including letters instead of words.

\section{Interference control}

\section{Delayed working memory paradigm}

This paradigm measures the ability to control interference from affectively distracting stimuli during working memory performance. ${ }^{73}$

On each trial, six capital letters are presented for 1500 $\mathrm{ms}$ and participants are asked to memorise them. The presentation of the letters is followed by a delay period of $2000 \mathrm{~ms}$, and the presentation of another single letter. Participants have to decide whether or not the single letter was part of the initial block of letters. During the delay period, participants are either presented with a blank screen, a neutral or a negative picture. All picture stimuli are taken from the International Affective Picture System (IAPS) ${ }^{74}$ Neutral and negative IAPS stimuli are matched for social content and perceptual complexity.

The ability to control interference from irrelevant information will be examined by comparing response latencies between blank screens, neutral and negative stimuli presented in the delay period.

\section{Stroop paradigm}

The Stroop task is based on a paradigm by Etkin and colleagues $^{75}$ and measures the ability to control interference from simultaneously presented irrelevant information.

Each trial consists of the presentation of a happy or an angry facial expression with the word 'Anger' or 'Happiness' printed across the facial expression. Participants are asked to ignore the words and to indicate by button press whether the face picture displays an angry or happy facial expression. Facial expressions and words are either congruent or incongruent. Each picture/word combination is presented for $1000 \mathrm{~ms}$. All facial expressions are taken from the original Ekman faces set. ${ }^{77}$
The ability to control interference from irrelevant information is assessed by the classical behavioural interference effect (ie, response latencies to incongruent trials as compared with response latencies to congruent trials). In addition, trials can be classified based on the congruence of the previous trial: congruent trial following a congruent trial (cC), incongruent trial following a congruent trail (cI), congruent trial following an incongruent trial (iC) and incongruent trial following an incongruent trial (iI).

\section{Additional measures}

Prediction and recall of affect, sleep and affect regulation strategies

In the first laboratory session, participants will be presented with all items from the ambulatory assessment (eg, affect, affect regulation strategies, sleep) and asked to indicate on a scale from 0 (not at all) to 7 (very much) how much they expect to feel or behave this way (on average) during the following 7 days. At the end of the ambulatory assessment period, participants will be presented with all items from the ambulatory assessment again and asked to indicate on a scale from 0 (not at all) to 7 (very much) how much they had felt or behaved this way (on average) during the past 7 days.

\section{Intelligence}

Participants' intelligence will be estimated by assessing the subtest 4 of the Leistungsprüfsystem (LPS-4) ${ }^{78}$ This serves to control for group differences in basic cognitive capabilities when examining group differences in cognitive control.

\section{Electrocardiogram}

At the end of the second laboratory session, participants will be asked to put on an ECG chest belt to measure their resting state heart rate variability for a $5 \mathrm{~min}$ period. Participants are asked to relax during the ECG assessment.

\section{Movement}

During the 7-day ambulatory assessment period, participants will be asked to wear an accelerometer attached to their hips. The accelerometer continuously assesses data regarding participants' acceleration in all three geometric axes, context temperature and air pressure. ${ }^{79}$ This will allow to examine individual levels of physical activity and energy expenditure during the ambulatory assessment period.

\section{Procedures}

The procedure of this project is depicted in figure 1. Data collection started in January 2017 and will last until the end of 2018.

\section{Sample size determination}

Power analysis for group differences in cognitive control

Previous studies examining impairments in valence-dependent cognitive control in depressed compared with control participants yielded medium between-group effect sizes (WMST task: eg, $\mathrm{d}=0.78$ ) ${ }^{38}$ Similar effect sizes 


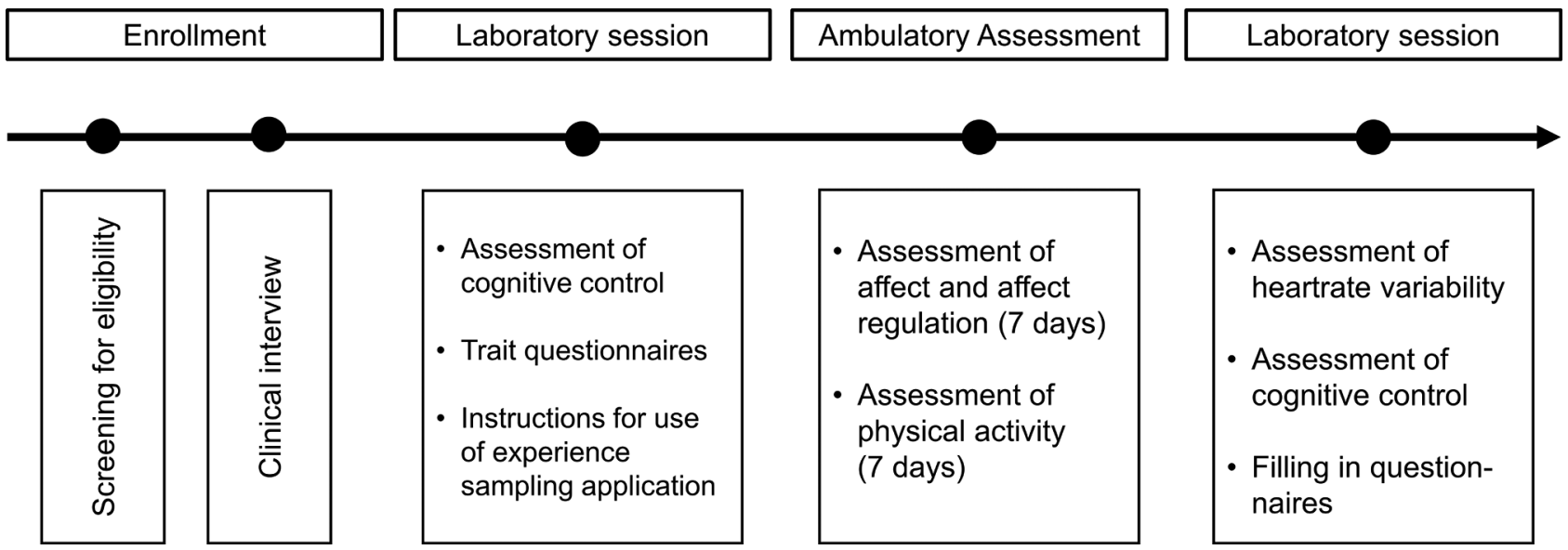

Figure 1 Procedure of the research project.

were obtained for group differences in valence-dependent cognitive control between individuals with BPD and healthy controls (interference control: eg, $\mathrm{d}=0.89$ ). ${ }^{34}$ To detect medium-sized group differences in cognitive control functions using univariate analyses of variance, a total sample size of $n=159$ ( $n=53$ per group) is needed as determined using $G^{*}$ Power (assuming $\alpha=0.05$, power of 0.8 ). For selected post hoc group comparisons, a group size of $\mathrm{n}=51$ is required (assuming $\alpha=0.05$, power of 0.8 , allocation ratio $=1$ ). Note that we will use multilevel modelling to test group differences in cognitive control. Given that multilevel modelling includes several assessment points per individual, the intended sample size of $n=159$ will be more than sufficient to detect medium-sized group differences in cognitive control using multilevel modelling.

\section{Power analysis for ambulatory assessment data}

A sample size of $n=159$ that is required for the experimental part of the study is also large enough to ensure appropriate power for analysing the ambulatory assessment data. For the ambulatory assessment part, all variables will be assessed eight times a day for seven consecutive days. For the multilevel analysis, this would mean that there are 56 occasions of measurement nested within 159 individuals resulting in 8904 data points. For variable affective states, it is reasonable to assume an intraclass correlation of 0.30 resulting in a design effect of $\mathrm{DE}=21.7{ }^{80}$ Therefore, our multilevel analysis would be approximately comparable to a classical multiple regression analysis with 410 individuals. ${ }^{80}$ Given this sample size, it would be possible to detect a small interaction effect between two independent variables (partial $R^{2}=0.01$ ) in a multiple regression analysis with a power of 0.8 (assuming $\alpha=0.05)$.

\section{Data analysis}

\section{Affective dynamics}

To assess whether groups differ in their affective dynamics (ie, inertia, instability, variability), we will calculate three different within-person measures for both positive and negative affect ${ }^{81}$ : (1) affective variability will be assessed by the within-subject SD of the respective affect scale. (2) Affective instability will be calculated as the within-subject root mean square successive difference between consecutive affect measures. (3) Inertia will be assessed by the temporal dependency of consecutive affect measures, that is, the within-subject lag-one autocorrelation. Autocorrelations will be Fisher's $\mathrm{z}$ transformed to normalise their distribution.

Based on the distribution of the respective indices, we will select adequate methods for testing group differences. We will further examine whether controlling for mean levels of positive or negative affect will have an impact on the main effect of Group. ${ }^{82}$

\section{Group differences in the use and effectiveness of affect regulation} strategies

To examine whether groups differ in the frequency of using rumination, suppression or reappraisal, we will employ hierarchical linear modelling. The dependent variable will be the intensity rating of the respective AR strategy assessed at time $t$. The predictor variable of interest will be Group (BPD, MDD, CTL). In addition, we will enter the intensity rating of all other strategies to control for overall AR strategy use.

To examine whether the effectiveness of rumination, suppression or reappraisal differs among groups, we will employ hierarchical linear modelling. The dependent variable will be the respective affect rating (positive or negative) at time $t$. Affect ratings at time $t-1$ will be entered as predictor. Predictor variables of main interest will be Group (BPD, MDD, CTL) and the intensity of each assessed AR strategy (reappraisal, rumination, suppression, distraction, acceptance, social sharing) employed between time $t-1$ and time $t$.

\section{Group differences in cognitive control functions}

Reaction time data from the behavioural experiments will be cleansed according to the following procedures: outliers in response latencies will be defined as values below or above the upper or lower fences of each individual's distribution in each experimental condition. Outliers will be eliminated. In addition, participants will be excluded from analyses if their overall accuracy level indicates that the task was not sufficiently understood. 
In a first step, group differences in cognitive control functions will be analysed using separate multilevel models per experiment. Response latency will be the dependent variable. The Experimental Condition, Stimuli Valence (where applicable) and Group will be entered as predictor variables.

In a second step, composite scores for the ability to discard irrelevant information from working memory and for the ability to control interference from distracting information will be generated. Group differences on these composite scores will be examined by using multilevel models. The respective composite score will be the dependent variable. Stimuli Valence and Group will be entered as predictor variables.

\section{Relating cognitive control functions and affect regulation}

To examine whether the use of rumination, suppression or reappraisal will be related to individual differences in cognitive control functions, we will employ hierarchical linear modelling. The dependent variable will be the intensity rating of the AR strategies assessed at time $t$. The predictor variables of interest will be the specific Strategy (reappraisal, rumination, suppression, distraction, acceptance, social sharing) and the Cognitive Control scores, as detailed below.

To examine whether the effectiveness of reappraisal, rumination or suppression will be related to individual differences in cognitive control functions, we will also employ hierarchical linear modelling. The dependent variable will be the respective affect rating (positive or negative) at time $t$. Affect ratings at time $t-1$ will be entered as predictor variable (see Group differences in the use and effectiveness of affect regulation strategies section). Further predictor variables of interest will be Group (BPD, MDD, CTL), the Cognitive Control scores (see below) and the Intensity of each assessed AR strategy (reappraisal, rumination, suppression, distraction, acceptance, social sharing) employed between time $t-1$ and time $t$.

Cognitive control indices for each experiment will be computed as follows: For the 'Working Memory Selection Task', the discarding index will be computed as the median response latency to suppress probes minus the median response latency to novel probes of the same valence. Two separate difference scores, one for each valence condition (positive, negative), will be computed.

For the RUT, the removal index will be assessed as the difference in response latencies between trials with short and long CTIs. This difference will be computed as a proportional gain score accounting for general processing speed (ie, Removal Speed $=($ mean $($ short CTI $)-$ mean (long CTI) )/mean (short CTI)). We will calculate two separate removal time indices for the removal of negative and positive words, respectively.

For the Stroop Task, the classical behavioural interference effect (ie, response latencies to incongruent trials minus response latencies to congruent trials) will be computed.
In the 'Delayed Working Memory Task', a general distraction score will be computed by subtracting response latencies in trials with blank screens presented in the delay period from response latencies in trials with neutral and negative IAPS stimuli presented in the delay period. In addition, we will calculate an 'affective distraction score' by subtracting response latencies for neutral stimuli from response latencies for negative stimuli.

\section{Patients and public involvement}

There was no further involvement of patients or the public in the development of this study protocol. The results of this study will be forwarded to interested participants. Results will be disseminated to relevant psychotherapeutic and patient communities in peer-reviewed journals, and at scientific conferences.

\section{Ethics and dissemination}

The study will be conducted in accordance with the Helsinki Declaration. The research team members have made sure that the study respects the following ethical principles: all the personal data gathered will be treated confidentially, written informed consent will be collected, data will be securely stored and the data will only be used for research purposes. Participation in this research study is voluntary. Participants will be reminded of their rights to withdraw from the study without giving any reason. Data privacy will be guaranteed: all the research data gathered during the project will be identified using pseudonyms. Personal data will be kept under lock and is stored separately from research data. Communications and publications will not enable identification of individual participants. Ambulatory assessment will be realised with a smartphone application (movisensXS) on devices provided by the research team. No further information of the participants' behaviour (eg, GPS tracking of movement profiles) is stored.

We plan to publish several articles in peer-reviewed scientific journals. In addition, we will communicate the results at scientific congresses. This research project will also result in a $\mathrm{PhD}$ thesis.

Contributors LS and UZ designed the study, wrote the funding grant, and drafted and revised the current manuscript. P-CB was responsible for the data analytic plan, wrote the section data analysis and revised the current manuscript. JB piloted the Removal and Updating task, coordinated the data assessment, and wrote and revised the current manuscript.

Funding Funding for this study was provided by grants from the German Research Foundation (DFG-ZE 1029/2-1 to UZ and DFG-SCHU 2961/2-1 to LS).

Competing interests None declared.

Patient consent Not required.

Ethics approval Ethics committee of Freie Universität Berlin, Germany (no. 67/2013; Amendment: 136/2017).

Provenance and peer review Not commissioned; externally peer reviewed.

Open access This is an open access article distributed in accordance with the Creative Commons Attribution Non Commercial (CC BY-NC 4.0) license, which permits others to distribute, remix, adapt, build upon this work non-commercially, and license their derivative works on different terms, provided the original work is properly cited, appropriate credit is given, any changes made indicated, and the use is non-commercial. See: http://creativecommons.org/licenses/by-nc/4.0/. 


\section{REFERENCES}

1. Linehan MM, Bohus M, Lynch TR. Dialectical behavior therapy for pervasive emotion dysregulation: theoretical and practical underpinnings. Gross JJ, ed. Handbook of emotion regulation. New York, NY US: Guilford Press, 2007:581-605.

2. Nolen-Hoeksema S, Wisco BE, Lyubomirsky S. Rethinking rumination. Perspect Psychol Sci 2008;3:400-24.

3. Joormann J, D'Avanzato C. Emotion regulation in depression: examining the role of cognitive processes. Cognition and Emotion 2010;24:913-39.

4. Ochsner KN, Gross JJ. The cognitive control of emotion. Trends Cogn Sci 2005;9:242-9.

5. Koval P, Kuppens P, Allen NB, et al. Getting stuck in depression: the roles of rumination and emotional inertia. Cogn Emot 2012;26:1412-27.

6. Bylsma LM, Morris BH, Rottenberg J. A meta-analysis of emotional reactivity in major depressive disorder. Clin Psychol Rev 2008;28:676-91.

7. Aldao A, Nolen-Hoeksema S, Schweizer S. Emotion-regulation strategies across psychopathology: a meta-analytic review. Clin Psychol Rev 2010;30:217-37.

8. Nolen-Hoeksema S, Morrow J. A prospective study of depression and posttraumatic stress symptoms after a natural disaster: the 1989 Loma Prieta Earthquake. J Pers Soc Psychol 1991;61:115-21.

9. Watkins ER. Constructive and unconstructive repetitive thought. Psychol Bull 2008;134:163-206.

10. Ehring T, Tuschen-Caffier B, Schnülle J, et al. Emotion regulation and vulnerability to depression: spontaneous versus instructed use of emotion suppression and reappraisal. Emotion 2010;10:563-72.

11. Quigley L, Dobson KS. An examination of trait, spontaneous and instructed emotion regulation in dysphoria. Cogn Emot 2014;28:622-35.

12. D'Avanzato C, Joormann J, Siemer M, et al. Emotion regulation in depression and anxiety: examining diagnostic specificity and stability of strategy use. Cognitive Ther Res 2013;37:968-80.

13. Gross JJ, John OP. Individual differences in two emotion regulation processes: implications for affect, relationships, and well-being. J Pers Soc Psychol 2003;85:348-62.

14. McRae K, Jacobs SE, Ray RD, et al. Individual differences in reappraisal ability: links to reappraisal frequency, well-being, and cognitive control. J Res Personality 2012;46:2-7.

15. $\mathrm{Pe} M L$, Raes F, Koval $\mathrm{P}$, et al. Interference resolution moderates the impact of rumination and reappraisal on affective experiences in daily life. Cogn Emot 2013;27:492-501.

16. Joormann J, Siemer M, Gotlib IH. Mood regulation in depression: differential effects of distraction and recall of happy memories on sad mood. J Abnorm Psychol 2007;116:484-90.

17. Troy AS, Wilhelm FH, Shallcross AJ, et al. Seeing the silver lining: cognitive reappraisal ability moderates the relationship between stress and depressive symptoms. Emotion 2010;10:783-95.

18. Stiglmayr CE, Grathwol T, Linehan MM, et al. Aversive tension in patients with borderline personality disorder: a computer-based controlled field study. Acta Psychiatr Scand 2005;111:372-9.

19. Ebner-Priemer UW, Kuo J, Kleindienst N, et al. State affective instability in borderline personality disorder assessed by ambulatory monitoring. Psychol Med 2007;37:961-70.

20. Schulze L, Domes G, Krüger A, et al. Neuronal correlates of cognitive reappraisal in borderline patients with affective instability. Biol Psychiatry 2011;69:564-73.

21. Schulze L, Schmahl C, Niedtfeld I. Neural correlates of disturbed emotion processing in borderline personality disorder: a multimodal meta-analysis. Biol Psychiatry 2016;79:97-106.

22. Beblo T, Fernando S, Kamper $\mathrm{P}$, et al. Increased attempts to suppress negative and positive emotions in borderline personality disorder. Psychiatry Res 2013;210.

23. Chapman AL, Specht MW, Cellucci T. Borderline personality disorder and deliberate self-harm: does experiential avoidance play a role? Suicide Life Threat Behav 2005;35:388-99.

24. Abela JR, Payne AV, Moussaly N. Cognitive vulnerability to depression in individuals with borderline personality disorder. $J$ Pers Disord 2003;17:319-29.

25. Baer RA, Sauer SE. Relationships between depressive rumination, anger rumination, and borderline personality features. Personal Disord 2011;2:142-50.

26. Chapman AL, Dixon-Gordon KL, Waiters K N. Borderline personality features moderate emotion reactivity and emotion regulation in response to a fear stresssor. J Experi Psychopathol 2013:1-20.

27. Chapman AL, Rosenthal MZ, Leung DW. Emotion suppression in borderline personality disorder: an experience sampling study. J Pers Disord 2009;23:29-47.
28. Svaldi J, Dorn C, Matthies S, et al. Effects of suppression and acceptance of sadness on the urge for non-suicidal self-injury and self-punishment. Psychiatry Res 2012;200:404-16

29. Koenigsberg HW, Fan J, Ochsner KN, et al. Neural correlates of the use of psychological distancing to regulate responses to negative social cues: a study of patients with borderline personality disorder. Biol Psychiatry 2009;66:854-63.

30. Lang S, Kotchoubey B, Frick C, et al. Cognitive reappraisal in trauma-exposed women with borderline personality disorder. Neuroimage 2012;59:1727-34.

31. Friedman NP, Miyake A. The relations among inhibition and interference control functions: a latent-variable analysis. J Exp Psychol Gen 2004;133:101-35.

32. Miyake A, Friedman NP. The nature and organization of individual differences in executive functions: four general conclusions. Curr Dir Psychol Sci 2012;21:8-14.

33. Domes G, Winter B, Schnell K, et al. The influence of emotions on inhibitory functioning in borderline personality disorder. Psychol Med 2006;36:1163-72.

34. Krause-Utz A, Oei NY, Niedtfeld I, et al. Influence of emotional distraction on working memory performance in borderline personality disorder. Psychol Med 2012;42:2181-92.

35. Prehn K, Schulze L, Rossmann S, et al. Effects of emotional stimuli on working memory processes in male criminal offenders with borderline and antisocial personality disorder. World J Biol Psychiatry 2013;14:71-8.

36. Wingenfeld K, Mensebach C, Rullkoetter N, et al. Attentional bias to personally relevant words in borderline personality disorder is strongly related to comorbid posttraumatic stress disorder. $J$ Pers Disord 2009;23:141-55.

37. Goeleven E, De Raedt R, Baert S, et al. Deficient inhibition of emotional information in depression. J Affect Disord 2006;93:149-57.

38. Joormann J, Gotlib IH. Updating the contents of working memory in depression: interference from irrelevant negative material. J Abnorm Psychol 2008;117:182-92.

39. Joormann J, Levens SM, Gotlib IH. Sticky thoughts: depression and rumination are associated with difficulties manipulating emotional material in working memory. Psychol Sci 2011;22:979-83.

40. Lau MA, Christensen BK, Hawley LL, et al. Inhibitory deficits for negative information in persons with major depressive disorder. Psychol Med 2007;37:1249-59.

41. Zetsche U, D'Avanzato C, Joormann J. Depression and rumination: relation to components of inhibition. Cogn Emot 2012;26:758-67.

42. Joormann J, Gotlib IH. Emotion regulation in depression: relation to cognitive inhibition. Cogn Emot 2010;24:281-98.

43. Gul A, Ahmad $\mathrm{H}$. Cognitive deficits and emotion regulation strategies in patients with psychogenic nonepileptic seizures: a task-switching study. Epilepsy Behav 2014;32:108-13.

44. Pe ML, Raes F, Kuppens P. The cognitive building blocks of emotion regulation: ability to update working memory moderates the efficacy of rumination and reappraisal on emotion. PLoS One 2013;8.

45. Baer RA, Peters JR, Eisenlohr-Moul TA, et al. Emotion-related cognitive processes in borderline personality disorder: a review of the empirical literature. Clin Psychol Rev 2012;32:359-69.

46. Donaldson C, Lam D. Rumination, mood and social problem-solving in major depression. Psychol Med 2004;34:1309-18.

47. Epp AM, Dobson KS, Dozois DJ, et al. A systematic meta-analysis of the Stroop task in depression. Clin Psychol Rev 2012;32:316-28.

48. Thayer JF, Lane RD. A model of neurovisceral integration in emotion regulation and dysregulation. J Affect Disord 2000;61:201-16.

49. Williams DP, Cash C, Rankin C, et al. Resting heart rate variability predicts self-reported difficulties in emotion regulation: a focus on different facets of emotion regulation. Front Psychol 2015;6:261.

50. Koval $P$, Ogrinz B, Kuppens $P$, et al. Affective instability in daily life is predicted by resting heart rate variability. PLoS One 2013;8:e81536.

51. Kemp AH, Quintana DS, Gray MA, et al. Impact of depression and antidepressant treatment on heart rate variability: a review and metaanalysis. Biol Psychiatry 2010;67:1067-74

52. Koenig J, Kemp AH, Feeling NR, et al. Resting state vagal tone in borderline personality disorder: a meta-analysis. Prog Neuropsychopharmacol Biol Psychiatry 2016;64:18-26.

53. Reed J, Buck S. The effect of regular aerobic exercise on positive-activated affect: a meta-analysis. Psychol Sport Exer 2009;10:581-94.

54. Kanning MK, Ebner-Priemer UW, Schlicht WM. How to investigate within-subject associations between physical activity and momentary affective states in everyday life: a position statement based on a literature overview. Front Psychol 2013;4:187.

55. Wenze SJ, Gunthert KC, German RE. Biases in affective forecasting and recall in individuals with depression and anxiety symptoms. Pers Soc Psychol Bull 2012;38:895-906. 
56. Loewenstein G. Affect regulation and affective forecasting. New York City: Guilford Press, 2007:180-203.

57. Levine LJ, Schmidt S, Kang HS, et al. Remembering the silver lining: reappraisal and positive bias in memory for emotion. Cogn Emot 2012;26:871-84.

58. Wittchen H-U, Wunderlich U, Gruschwitz S, et al. SKID-I Strukturiertes Klinisches Interview für DSM-IV. Achse I: Psychische Störungen. Interviewheft. Göttingen: Hogrefe, 1997.

59. Fydrich T, Renneberg B, Schmitz B, et al. In: Michael B ed, SKID-II: strukturiertes klinisches Interview für DMS-IV; Achse II: Persönlichkeitsstörungen; Interviewheft; eine deutschsprachige, erw. Bearb. der amer. Originalversion des SKID-II von. Göttingen: Hogrefe, 1997.

60. Beck AT, Steer RA, Brown GK. Manual for the Beck Depression Inventory-II. San Antonio, TX: Psychological Corporation, 1996.

61. Kühner $\mathrm{C}$, Bürger $\mathrm{C}$, Keller $\mathrm{F}$, et al. Reliabilität und Validität des revidierten Beck-Depressionsinventars (BDI-II). Nervenarzt 2007;78:651-6.

62. Bohus M, Kleindienst N, Limberger MF, et al. The short version of the Borderline Symptom List (BSL-23): development and initial data on psychometric properties. Psychopathol 2009;42:32-9.

63. Derogatis LR. SCL-90-R. Administration, scoring and procedures Manual-II. 2nd edn. Baltimore: Clinical Psychometric Research, 1983.

64. Geisheim C, Hahlweg K, Fiegenbaum W, et al. Das Brief Symptom Inventory (BSI) als Instrument zur Qualitätssicherung in der Psychotherapie. Diagnostica 2002;48:28-36.

65. Huffziger S, Kühner C. Die Ruminationsfacetten brooding und reflection. Zeitschrift für Klinische Psychologie und Psychotherapie 2012;41:38-46.

66. Watson D, Clark LA, Tellegen A. Development and validation of brief measures of positive and negative affect: the PANAS scales. $J$ Pers Soc Psychol 1988;54:1063-70.

67. Stiglmayr C, Schmahl C, Bremner JD, et al. Development and psychometric characteristics of the DSS-4 as a short instrument to assess dissociative experience during neuropsychological experiments. Psychopathology 2009:42:370-4.

68. Heiy JE, Cheavens JS. Back to basics: a naturalistic assessment of the experience and regulation of emotion. Emotion 2014:14:878-91.
69. Zhang JX, Leung HC, Johnson MK. Frontal activations associated with accessing and evaluating information in working memory: an fMRI study. Neuroimage 2003;20:1531-9.

70. Võ ML, Conrad M, Kuchinke L, et al. The Berlin Affective Word List Reloaded (BAWL-R). Behav Res Methods 2009;41:534-8.

71. Ecker UKH, Lewandowsky S, Oberauer K. Removal of information from working memory: a specific updating process. J Memory Language 2014;74:77-90.

72. Chang EP, Ecker UKH, Page AC. Impaired memory updating associated with impaired recall of negative words in dysphoric rumination-evidence for a removal deficit. Behav Res Ther 2017;93:22-8.

73. Oei NY, Tollenaar MS, Spinhoven P, et al. Hydrocortisone reduces emotional distracter interference in working memory. Psychoneuroendocrinology 2009;34:1284-93.

74. Lang PJ, Bradley MM, Cuthbert BN. International affective picture system (IAPS): affective ratings of pictures and instruction manual. Gainesville, FL: University of Florida, 2008.

75. Etkin A, Egner T, Peraza DM, et al. Resolving emotional conflict: a role for the rostral anterior cingulate cortex in modulating activity in the amygdala. Neuron 2006;51:871-82.

76. Etkin A, Prater KE, Hoeft $F$, et al. Failure of anterior cingulate activation and connectivity with the amygdala during implicit regulation of emotional processing in generalized anxiety disorder. Am J Psychiatry 2010;167:545-54.

77. Ekman P. Pictures of facial affect: Consulting Psychologists Press, 1976.

78. Horn W. L-P-S Leistungsprüfsystem. Göttingen: Hogrefe, 1983.

79. Hey S, Anastasopoulou P, von Haaren B. Erfassung körperlicher Aktivität mittels Akzelerometrie-Möglichkeiten und Grenzen aus technischer Sicht. B\&G Bewegungstherapie und Gesundheitssport 2014;30:73-8.

80. Eid M, Gollwitzer M, Schmitt M. Statistik und Forschungsmethoden Weinheim: Beltz PVU, 2013.

81. Koval P, Pe ML, Meers K, et al. Affect dynamics in relation to depressive symptoms: variable, unstable or inert? Emotion 2013;13:1132-41.

82. Ebner-Priemer UW, Eid M, Kleindienst N, et al. Analytic strategies for understanding affective (in)stability and other dynamic processes in psychopathology. J Abnorm Psychol 2009;118:195-202. 